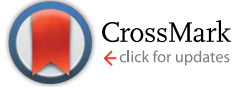

Cite this: RSC Adv., 2016, 6, 92197

Received 20th July 2016

Accepted 20th September 2016

DOI: $10.1039 / c 6 r a 18483 a$

www.rsc.org/advances

\section{A superrepellent coating with dynamic fluorine chains for frosting suppression: effects of polarity, coalescence and ice nucleation free energy barrier}

\author{
Takeo Moriya, ${ }^{a}$ Kengo Manabe, ${ }^{a}$ Mizuki Tenjimbayashi, ${ }^{a}$ Ken Suwabe, ${ }^{a}$ \\ Hirotaka Tsuchiya, ${ }^{a}$ Takeshi Matsubayashi, ${ }^{a}$ Walter Navarrini ${ }^{\mathrm{b}}$ and Seimei Shiratori ${ }^{\star a}$
}

Ice formation on surfaces is a serious issue in many different fields in terms of function, safety, and cost of operation in human life. Hydrophobic coating technology is one of the effective ways to prevent ice formation. Previous studies focused on the effects of surface structure and surface chemical modification on anti-icing ability. However, only a few studies have clarified a method to inhibit the initial formation of ice on surfaces; in addition, an effective mechanism for anti-frosting has not been identified as yet. Here, hydrophobic smooth surface coatings using three coupling agents with low surface energy and different molecular chain dynamics were fabricated. The surface roughnesses were lower than $1 \mathrm{~nm}$. The fluorocarbon-based coatings delayed frost formation compared with the uncoated surface until $-6{ }^{\circ} \mathrm{C}$. We explored why the coating surface prevented frost formation and the effects of surface chemical modification on frost resistance from the viewpoint of heat exchange contact area during droplet coalescence, ice nucleation free energy barrier, polarity and polarizability of the coated surface.

\section{Introduction}

Damage induced by freezing has tended to increase because of localized cold phenomena caused by abnormal weather patterns. Icing and frosting of surfaces are also inconvenient in our daily life. For example, icing on exposed surfaces leads to operational difficulties and high maintenance effort for power networks, aircraft, ships, and ground transportation vehicles. $^{1-12}$ Although there are some anti-icing systems currently available that operate through electrothermal, ${ }^{\mathbf{1 , 7}}$ chemical, $^{\mathbf{1 , 5 , 6}}$

${ }^{a}$ Center for Material Design Science, School of Integrated Design Engineering, Keio University, 3-14-1 Hiyoshi, Kohoku-ku, Yokohama, Kanagawa 223-8522, Japan. E-mail: shiratori@appi.keio.ac.jp; Fax: +81-45-566-1602; Tel: +81-45-566-1602 ${ }^{b}$ Politecnico di Milano, Dipartimento di Chimica, Materiali e Ingegneria Chimica, Via Mancinelli 7, 20131 Milano, Italy

$\dagger$ Electronic supplementary information (ESI) available: The chemical structure of decyltrimethoxysilane (DTMS), tridecafluoro-1,1,2,2-tetrahydrooctyl-triethoxysilane (FAS13) and heptadecafluoro-1,1,2,2-tetrahydrodecyl-triethoxy-silane (FAS17), FE-SEM image, FT-IR spectra, film thickness distribution measured by ellipsometry analysis, transmittance measured by a spectrophotometer, total transmittance (TT), parallel transmittance (PT), diffusion (DIF) and haze values (HAZE) measured by a haze meter, the polarity factors of water, formamide and hexadecane, the polarity factors of the coating surface, photographic images of heating surface after frosting test, the binarized photographic images during frosting test, the surface temperature with glass substrate and the coating surface, contact angle, sliding angle on the coating surface before freezing with after freezing, classic heterogeneous nucleation theory, Hückel charge distribution of alkyl chain, fluorocarbon and water molecular, the photographic images of frosting and water sliding angle of coating surfaces are changed molar ratio FAS to TEOS of solution, schematic image of measuring equipment of anti-frosting test. See DOI: 10.1039/c6ra18483a and mechanical deicing methods, ${ }^{\mathbf{1 , 4}, 7}$ most conventional antiicing and deicing strategies possess disadvantages from the viewpoints of energy consumption, cost, or environmental effects. $^{\mathbf{1 , 8}}$

Over the past couple of decades, many studies have focused on superhydrophobic surfaces, ${ }^{\mathbf{1 3 - 1 8}}$ which possess a water contact angle higher than $150^{\circ}$ and low water sliding angle, to delay or prevent ice formation. ${ }^{19-22}$ Superhydrophobic surfaces promote timely removal of water droplets before freezing

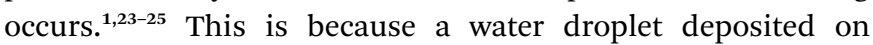
a superhydrophobic surface stays in the Cassie-Baxter state and has a large contact angle and small sliding, which lead to both minimal contact area and reduced contact time. ${ }^{\mathbf{1 , 2 , 1 9 - 2 2}}$ Despite the definite delay in freezing time of large size water droplets on superhydrophobic surfaces, small size water droplets rapidly freeze on this kind of surfaces which do not sufficiently repel small size water droplets (Fig. S1 $\dagger$ ). This can lead to structural collapse of superhydrophobic surfaces during repeated freezing and thawing cycles because the frost formed on the surface has nano/microscale texture..$^{\mathbf{9 , 1 0 , 2 6 - 2 9}}$

To overcome the above-mentioned challenges, T. S. Wong et al. have developed a slippery liquid-infused porous surface(s) (SLIPS). ${ }^{30-33}$ SLIPS is a film with nano/microstructured structures to immobilize a hydrophobic lubricant to repel targeting liquid which is immiscible with lubricant. ${ }^{30}$ SLIPS-coated substrates are frost repellent because the SLIPS easily removes the micro size condensed water. ${ }^{34-37}$ In addition, when the deposited water freezes, the immobilized lubricating oil in the SLIPS prevents penetration of frost into the surface texture and 
leads to a low ice adhesion strength. ${ }^{1,2,38}$ Although SLIPS-coated substrates are thought to be effective for frost repellent, ${ }^{34-37}$ Rykaczewski et al. indicated the limitation of SLIPS for antiicing that the lubricating oil is lost during each freezing and thawing cycle because lubricant is displaced by frozen water droplets allowing to penetrate the porous texture at low temperature. ${ }^{39}$

Thus, these approaches to produce anti-icing and antifrosting surfaces using hydrophobic coatings are limited by the rough texture of such coating surface, which allows penetration of condensed water, ice or frost. Moreover, the functionality of these coatings is deteriorated by repeated freezing and thawing cycles, when structural collapse or lubricant loss occurs. We have hypothesized that the following factors are important for realizing long-term anti-frosting surfaces with hydrophobic approach. The first one is a smooth surface without rough structure, where frost does not tend to nucleate. Secondly, an anti-frost coating with hydrophobic approach requires hydrophobicity to enhance the nucleation free energy barrier ${ }^{\mathbf{4 0 , 4 1}}$ and prevent adhesion of condensed water droplets. Finally, the coating also requires high water droplet mobility to promote coalescence of condensed droplets ${ }^{37}$ because a smaller liquid-solid contact area minimizes the loss of thermal radiation..$^{\mathbf{4 0 , 4 1}}$ In addition, the polarity/polarizability effects between water molecules and surface molecules at freezing temperature has been discussed. The strong interaction between coated surface and water molecules lead to be difficult to be frozen. ${ }^{54}$ Moreover, some previous researches have reported a charge state of the surface material affects heterogeneous ice nucleation. ${ }^{\mathbf{4 2 , 4 3}}$ Considering these requirements, we aimed to prepare a hydrophobic smooth surface without lubricant to realize long-term antifrosting (Scheme 1).

In this work, we focus on a hydrophobic molecular chain tethered to the smooth surface that was reported in $2012 .^{\mathbf{4 4 - 4 7}}$ This surface can shed off both water and solvents because the hydrophobic molecular chains dynamically work as a lubricant layer of SLIPS. ${ }^{\mathbf{4 4 - 4 7}}$ We suppose that such a coated surface with high molecular mobility should overcome the limitations of superhydrophobic surfaces and SLIPS for stable anti-frosting. Previous studies have focused on understanding the effect of a specific morphological surface structure on anti-icing and anti-frosting properties..$^{23-25,48,49}$ However, no previous research that focuses on chemical effects of molecular chain on frosting resistance are reported to the best of our knowledge. We assume the effects of chemical surface modification on ice nucleation are important because surface properties such as surface tension and polarity may change by surface chemical modification. ${ }^{50-55}$

In this work, three kinds of hydrophobic smooth surfaces are prepared by coating glass substrates with three lowsurface-energy silane couplers, decyltrimethoxysilane (DTMS), tridecafluoro-1,1,2,2-tetrahydrooctyl triethoxysilane (FAS13), and heptadecafluoro-1,1,2,2-tetrahydrodecyl triethoxysilane (FAS17). We investigated the surface energy, slipping performance and anti-frosting properties of each sample. Moreover, we confirmed the reason why the coated

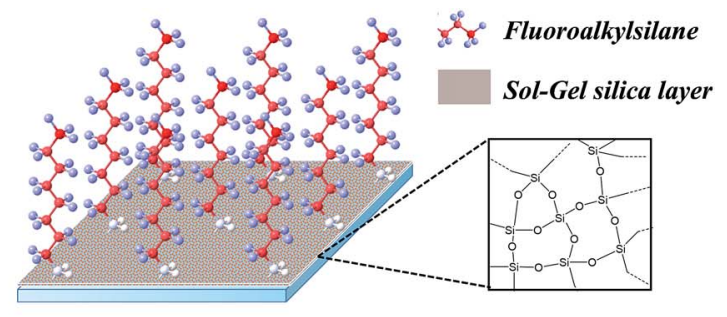

Concepts
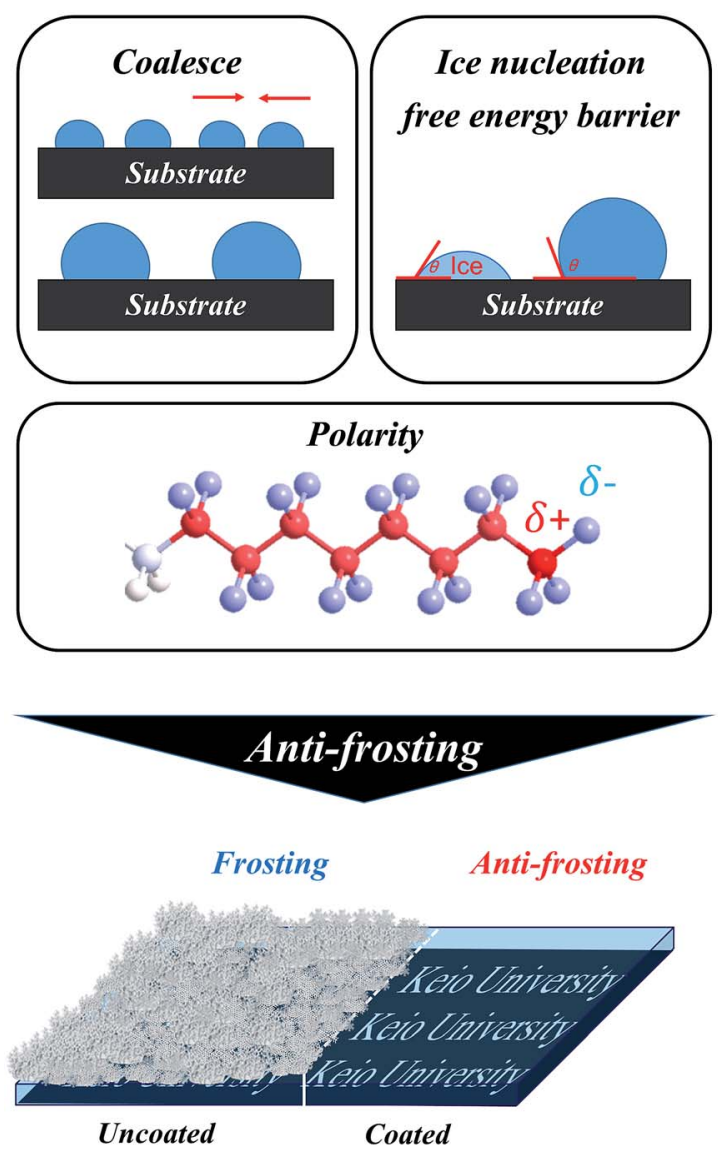

Scheme 1 Outline of our research concept. Superrepellent smooth surface with dynamic fluorine chains for anti-frosting were prepared. Three factors of delay frosting time on the coated and uncoated surface, which are coalesced condensed droplet to decrease contact area, high ice nucleation free energy barrier, and polarizability of fluorine material to decrease the mobility of water molecular.

surfaces prevent frost formation and the effect of chemical modification on the frost resistance behavior of the surfaces from various aspects, including the nucleation free energy barrier, surface-ice interfacial phenomenon and Hückel charge distribution of molecular chain. This research investigated the anti-frosting factor from the point of physical and chemical effect on coated surface and showed directions for the further development of anti-frosting coating technology. 


\section{Experimental}

\section{Materials}

DTMS was purchased from Shin-Etsu Chemical Co. Ltd. (Tokyo, Japan). FAS13 and FAS17 were obtained from Gelest Inc. (NC, USA). The chemical structures of DTMS, FAS13, and FAS17 are shown in the ESI (Fig. S2 $\dagger$ ). Tetraethoxysilane (TEOS) was purchased from Wako Pure Chemical Industry Ltd. (Osaka, Japan). Ethanol (EtOH; $\geq 99.5 \mathrm{wt} \%$ ) and hydrochloric acid ( $\mathrm{HCl}$; $\geq 30-35 \mathrm{wt} \%$ ) were purchased from Kanto Chemical Co. Inc. (Tokyo, Japan). An Aquarius GS-500.CPW (Advantec Co., Saijo, Japan) purifier was used to generate ultrapure water. Glass substrates $(76 \times 26 \mathrm{~mm})$ with a thickness of $1.0 \mathrm{~mm}$ and refractive index of 1.52 purchased from Matsunami Glass Ind. Ltd. (Kishiwada, Japan) were used to fabricate the films. The glass substrates were cleaned in potassium hydroxide $(\mathrm{KOH})$ solution (weight ratio of $\mathrm{KOH}: \mathrm{H}_{2} \mathrm{O}: \mathrm{EtOH}=1: 40: 60$ ) for 5 min, and then rinsed thoroughly with ultrapure water before use. KOH was obtained from Junsei Chemical Co. Ltd. (Tokyo, Japan). Formamide purchased from Tokyo Chemical Industry Co., Ltd. (Tokyo, Japan) and hexadecane from Wako Pure Chemical Industry, Ltd. were used to measure contact angle and surface energy.

\section{Surface coating formation}

The precursor solutions were prepared by mixing silane coupler (DTMS, FAS13 or FAS17) and TEOS in an EtOH/HCl solution for $24 \mathrm{~h}$ at room temperature. The typical molar ratio of the precursor solution was silane coupler: $9.54 \times 10^{-4} \mathrm{~mol}(0.25 \mathrm{~g}) /$ TEOS: $7.20 \times 10^{-3} \mathrm{~mol}(1.50 \mathrm{~g}) / \mathrm{EtOH}: 2.71 \times 10^{-1} \mathrm{~mol}(12.5 \mathrm{~g}) /$ $\mathrm{H}_{2} \mathrm{O}: 5.55 \times 10^{-2} \mathrm{~mol}(1.0 \mathrm{~g}) / \mathrm{HCl}: 7.7 \times 10^{-6} \mathrm{~mol}(0.94 \mathrm{mg})$. The glass substrates cleaned in $\mathrm{KOH}$ were dip-coated in the precursor solutions using a dipping speed of $4.0 \mathrm{~mm} \mathrm{~s}^{-1}$, immersion for $5 \mathrm{~s}$, and withdrawal at a speed of $4.0 \mathrm{~mm} \mathrm{~s}^{-1}$. The coated glass substrates were dried in air at room temperature for more than $24 \mathrm{~h}$ before characterization.

\section{Frosting test}

The glass substrates coated with DTMS, FAS13 and FAS17 were set on a Peltier element in a thermo-hygrostat at a relative humidity of $80 \%$ and temperature of $10^{\circ} \mathrm{C}$, and then the Peltier element was gradually cooled from $+10{ }^{\circ} \mathrm{C}$ to $-8 \pm 1{ }^{\circ} \mathrm{C}$. Photographic images were captured by a digital camera (PI400, Optris, USA) at intervals of $1 \mathrm{~min}$. After $60 \mathrm{~min}$ from the start, the unit was heated to $10{ }^{\circ} \mathrm{C}$ to examine frost removal ability (Fig. S3†).

In the same manner as the frosting tests, the samples were set on a Peltier element in air with a relative humidity of about $49 \%$ and temperature of $23{ }^{\circ} \mathrm{C}$. The Peltier element was gradually cooled from +10 to $-8 \pm 1{ }^{\circ} \mathrm{C}$. Photographic images were recorded by a CCD camera at intervals of $5 \mathrm{~min}$.

\section{Characterization}

Surface topography was observed using atomic force microscopy (AFM; Nanoscope IIIa, Digital Instruments, USA). The thickness and refractive index of the thin films coated on the glass substrates were determined by ellipsometry (MARY-102, Five Lab Co., Saitama, Japan). Surface modification was observed by X-ray photoelectron spectroscopy (XPS; JPS-9010 $\mathrm{TR}$, JEOL, Japan) with $\mathrm{Mg} \mathrm{K} \alpha$ radiation. The contact and sliding angles of $5 \mu \mathrm{L}$ droplets were measured using a contact angle meter (CA-DT, Kyowa, Tokyo, Japan). Contact angle hysteresis was measured by spreading and shrinking a droplet using a syringe pump set at a rate of $600 \mu \mathrm{L} \mathrm{min}^{-1}$ and optical microscope.

\section{Results and discussion}

\section{Surface characteristics}

We needed to fabricate a smooth uniform surface with liquidrepelling properties because ice nucleation is promoted on heterogeneous surfaces. The three surfaces prepared using DTMS, FAS13 and FAS17 were smooth and possessed low rootmean-square (RMS) roughnesses of less than $1 \mathrm{~nm}$ (Fig. S6† and Table 1). In addition, the ellipsometry analysis indicated that all surfaces were uniformly coated (Fig. S7†) and the film thickness was around $200 \mathrm{~nm}$ (Table 1). Therefore, the surfaces coatings prepared using DTMS, FAS13 and FAS17 have the same topological structure.

To confirm the modification of the substrates with lowsurface-energy agents, the prepared samples were evaluated by XPS. The surface coated with DTMS exhibited an intense C1s peak and that coated with FAS17 showed a more intense F1s peak than that coated with FAS13, as illustrated in Fig. 1a. The surface coated with DTMS possessed the strongest $-\mathrm{CH}_{2}-\mathrm{CH}_{2}-$ peak $(283.5 \mathrm{eV})$ of the three coated surfaces, while the spectra of surfaces coated with FAS13 or FAS17 contained a $-\mathrm{CF}_{2}-\mathrm{CF}_{2}-$ peak at $291.7 \mathrm{eV}$ (Fig. $1 \mathrm{~b}$ and c). ${ }^{56}$ The fluorine surface concentration of the FAS17 coating was about 1.3 times larger than that of the FAS13 one (Table $\mathrm{S} 1 \dagger$ ). These results confirm that these substrate surfaces were coated with low-surfaceenergy agents. Indeed the modified surfaces were oleophobic and hydrophobic. The sliding angle of water on the pristine surfaces was about $20^{\circ}$, while those of stearic acid and hexadecane were about $10^{\circ}$. The surface coated with DTMS tended to show lower sliding angles than those of surfaces coated with FAS13 or FAS17 (Fig. 2a). This is probably due to the higher mobility of DTMS compare to FAS13 and FAS17, indeed the water droplet mobility is influenced by the interaction between water molecules and the coated surface. ${ }^{55}$ The sliding angle of hexadecane and oleic acid which are organic liquid with low dielectric constant were lower than that of water because organic liquid is not influenced by interaction between surface

Table 1 RMS roughness measured by AFM and film thickness determined by ellipsometry

\begin{tabular}{lccc}
\hline & DTMS & FAS13 & FAS17 \\
\hline RMS roughness $(\mathrm{nm})$ & 0.360 & 0.646 & 0.414 \\
Film thickness $(\mathrm{nm})$ & 235.7 & 222.9 & 235.6
\end{tabular}


(a)

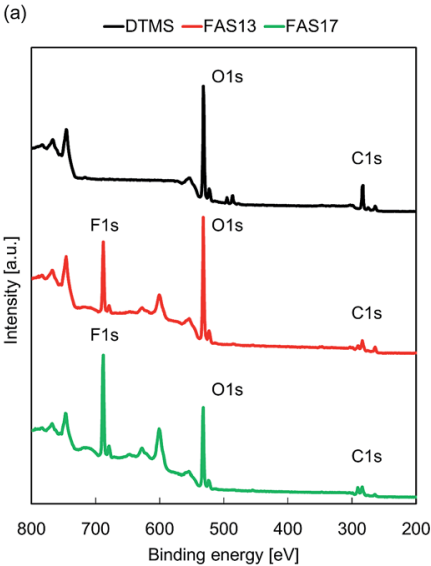

(b)

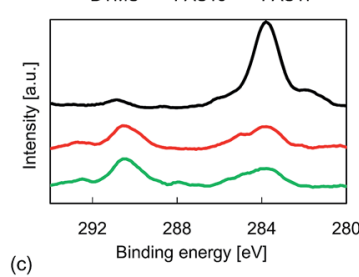

(c)

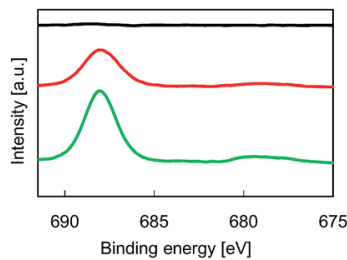

Fig. 1 XPS analyses of glass surfaces coated with DTMS, FAS13, and FAS17, respectively. (a) Survey spectra, (b) C1s core spectra, and (c) F1s core spectra.

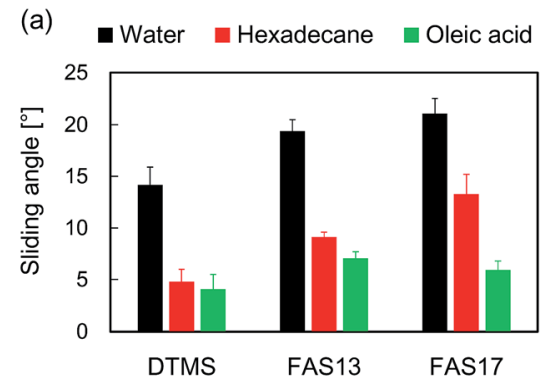

(b)

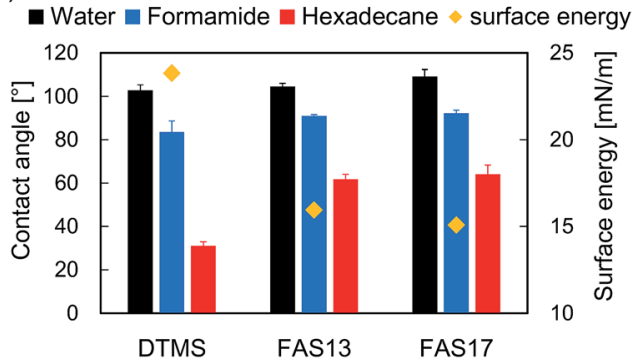

Fig. 2 Comparison of (a) sliding angles of water, hexadecane, oleic acid, and (b) contact angles of water, formamide and hexadecane and the surface energies of the surfaces coated with DTMS, FAS13 and FAS17 calculated using eqn (1)-(3).

molecular and organic liquid. Therefore, the sliding angle of oleic acid is influenced by the molecular chains with low surface energy, and the sliding angle of oleic acid on FAS13 is larger than FAS17. ${ }^{47}$ The contact angle of water and formamide were almost the same on all the surfaces. However, that of hexadecane on the surfaces coated with FAS13 or FAS17 was higher than that of the surface coated with DTMS (Fig. 2b). From this contact angle data, we calculated the surface energy of the samples using eqn (1)-(3). ${ }^{57}$

$$
(1+\cos \theta) \gamma_{\mathrm{L}}=2\left(\sqrt{\gamma_{\mathrm{s}}^{\mathrm{LW}} \gamma_{\mathrm{L}}^{\mathrm{LW}}}+\sqrt{\gamma_{\mathrm{s}}^{+} \gamma_{\mathrm{L}}^{-}}+\sqrt{\gamma_{\mathrm{s}}^{-} \gamma_{\mathrm{L}}^{+}}\right)
$$

$$
\begin{gathered}
\gamma_{\mathrm{s}}^{\mathrm{AB}}=2 \sqrt{\gamma_{\mathrm{s}}^{+} \gamma_{\mathrm{s}}^{-}} \\
\gamma_{\mathrm{s}}=\gamma_{\mathrm{s}}^{\mathrm{LW}}+\gamma_{\mathrm{s}}^{\mathrm{AB}}
\end{gathered}
$$

where $\gamma^{\mathrm{LW}}$ is the apolar component of surface tension (Lifshitz-van der Waals, LW), $\gamma^{\mathrm{AB}}$ is the polar component of surface tension (Lewis acid-base, $\mathrm{AB}$ ), $\gamma^{+}$is the electronacceptor parameter of the polar $\mathrm{AB}$ component, $\gamma^{-}$is the electron-donor parameter of the polar $\mathrm{AB}$ component, $\mathrm{L}$ is liquid, and $S$ is solid. The surface energy decreased from DTMS to FAS13 to FAS17 (Fig. 2b and Table S3†). These results indicate that the hydrophobic smooth surfaces fabricated in this study have different surface energy, the surfaces prepared with FAS13 and FAS17 are also oleophobic and possess similar surface energies.

\section{Anti-frosting properties of the coated surfaces}

We observed frost formation on the surfaces coated with DTMS, FAS13, and FAS17 using a digital camera (Fig. 3a). The uncoated glass substrate froze already $10 \mathrm{~min}$ after the start of the frosting test at $-8{ }^{\circ} \mathrm{C}$. In contrast, the coated glass substrates did not freeze after $10 \mathrm{~min}$ at $-8{ }^{\circ} \mathrm{C}$. After $15 \mathrm{~min}$ at this temperature, frost formation was observed on the surface coated with DTMS and after $20 \mathrm{~min}$, this surface was covered with frost (Fig. 3a). Frost formed on part of the surfaces coated with FAS13 and FAS17 after $20 \mathrm{~min}$. In particular, the FAS17 coating prevented frost from spreading. We measured the surface temperature by using thermography. Then, we confirmed the surface temperature has been constant value. Therefore, the influence of temperature variability cannot be a crucial factor, here (Fig. S10†).

Areas with frost cause light scattering because the frost contains many voids. Therefore, we calculated ice area fraction by using binarized photographic images obtained during the anti-frosting test at intervals of $10 \mathrm{~min}$ (Fig. $\mathrm{S} 12 \dagger$ ); the results are plotted in Fig. 3b. According to these results, the coatings on the substrates delayed frost formation, compared with that on an uncoated glass substrate. In addition, the spreading speed of frost formation depended on the agent used for surface modification (Fig. 3b).

We consider that three factors delay frosting time on the coated surfaces compared with that on an uncoated surface: (1) the contact area between the substrate and condensed droplet decreases as well as the area exposed to the humid atmosphere $(80 \%)$ present in the thermos-hydrostat (Fig. S3†), (2) the nucleation free energy barrier of the coated surface is higher than that of the uncoated glass substrate, and (3) the mobility of water molecules on the hydrophobic surface decreases because of the interaction with the modified surface, this fact is particularly evident with the fluorinated surface.

\section{Contact area decrease}

The contact area between the substrate and condensed droplet was reduced due to the coatings. According to magnified views of the surfaces during the frosting test, micro-sized droplets 
(a)

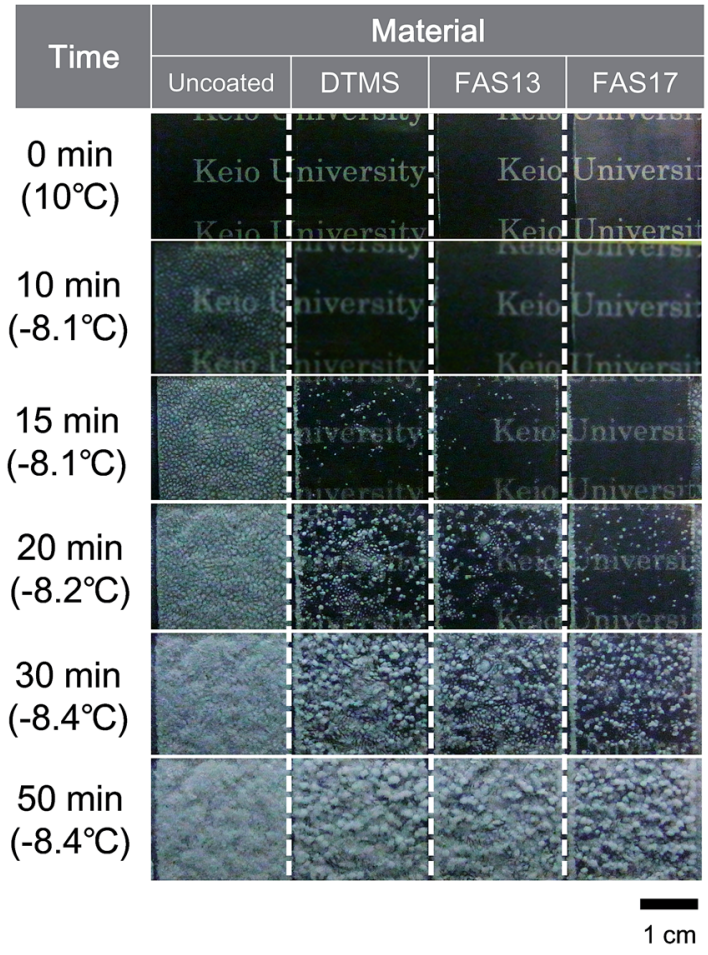

(b) $\rightarrow$ Uncoated $\rightarrow$ DTMS $\rightarrow$-FAS13 $\rightarrow$ FAS17

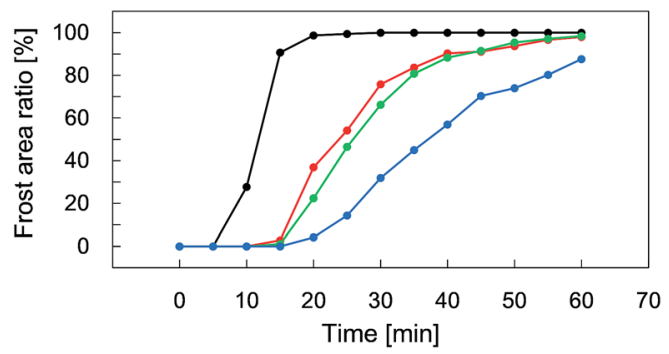

Fig. 3 (a) Photographic images of the surfaces during the frosting test recorded by a digital camera. The temperature was determined by the Peltier element. (b) Time dependence of the frost area ratio of surfaces coated with different agents.

grew on the cooled substrates because of water condensation and because of coalescence of little droplet to bigger droplets (Fig. 4a). On the uncoated substrate, condensed droplets covered the substrate because it was hydrophilic. In contrast, on coated surfaces condensed droplets did not cover the substrates because of the hydrophobicity of samples (Fig. 4a). The microsized condensed droplets coalesced each other to form large droplets on the surface coated in particular with DTMS (Fig. 4b) because the condensed droplets on this coated surface moved easily compared to the fluorinated surfaces (Fig. 2a). ${ }^{\mathbf{4 4 - 4 7}}$ And, DTMS coated surface was easy to adhere the water droplet from humidity because the surface energy of DTMS was larger than fluorinated surfaces. This can be proved geometrically because the contact area between a hydrophobic substrate and coalesced droplet is smaller than that between a hydrophilic substrate and the same volume of scattered droplets. (a)

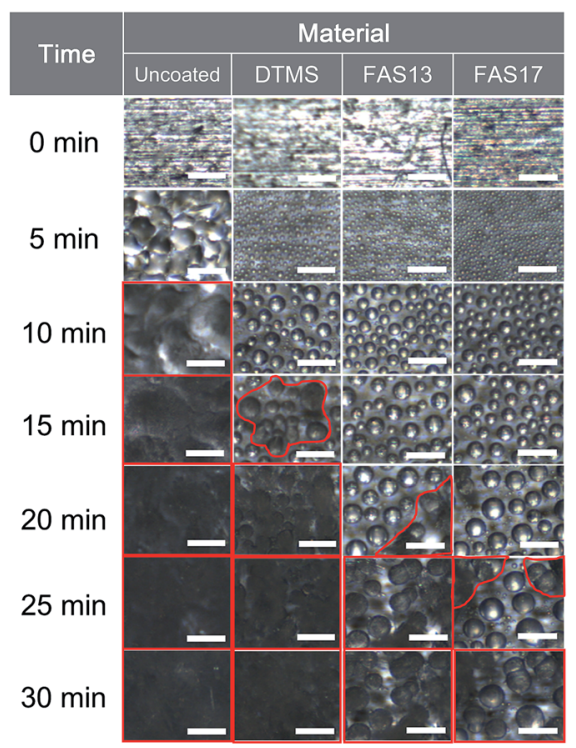

(b)

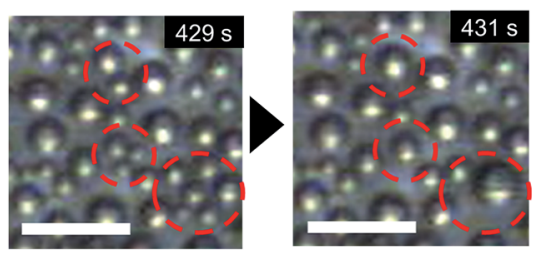

(c)

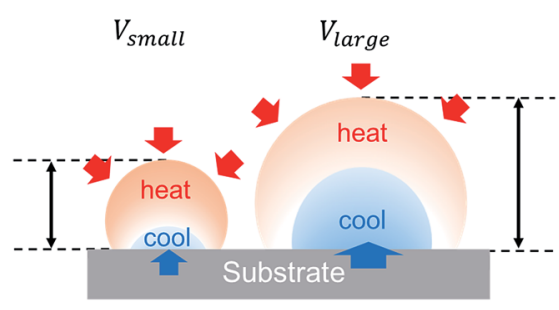

Fig. 4 (a) Enlarged images of the frosting test captured by a CCD camera. Condensed droplets surrounded by a red line are freezing. (b) Condensed droplets on the surface coated with FAS17 before coalescence (at $429 \mathrm{~s}$; left), and after coalescence (at $431 \mathrm{~s}$; right). The condensed droplets coalesced with each other to form larger droplets. The contact area between the solid surface and droplet decreased after coalescence. The scale bar is $500 \mu \mathrm{m}$. (c) Schematic image of heat flow in condensed droplet compared small size droplet with large size droplet.

$$
\begin{gathered}
s_{1}+s_{2}+\ldots+s_{n}=\sum_{i}^{n} s_{i}=\sum_{i}^{n} \pi\left(r_{i} \sin \theta\right)^{2} \\
S=\pi(R \sin \theta)^{2}=\pi(\sin \theta)^{2}\left(\sum_{i}^{n} r_{i}^{3}\right)^{\frac{2}{3}}
\end{gathered}
$$

where $s_{i}$ is the contact area between a scattered droplet and the substrate, and $S$ is the contact area between a coalesced droplet and substrate. In addition, $r_{i}$ is radius of curvature of the scattered water droplet, $R$ is the radius of curvature of a coalesced water droplet, and $\theta$ is the contact angle between a water droplet and solid surface. Here, to show that $\sum_{i}^{n} s_{i}$ is larger than $S$, we 
prove the following equation. Further details are described in the ESI. $\dagger$

$$
\sum_{i}^{n} r_{i}{ }^{2}>\left(\sum_{i}^{n} r_{i}^{3}\right)^{\frac{2}{3}}
$$

From the proof provided in the ESI, $\dagger$ we obtained the following relationship.

$$
\therefore \sum_{i}^{n} s_{i}>S
$$

Eqn (7) indicates that the contact area between a substrate and coalesced droplet was smaller than that between a substrate and the same volume of scattered droplets. These calculations indicate that the contact area between a condensed droplet and substrate is decreased by droplet coalescence on the hydrophobic surfaces. However, the effect of contact area between solid-liquid interface when condensed droplets volume became bigger.

Contemporarily to this decrement, also the ratio between droplet surface area exposed to humidity and volume of the droplet decreases, these two opposing effects regulate the heat flow across the droplet therefore also the effective droplet temperature. Thus, we observe a less efficient droplet heating from the gas phase and cooling from the substrate (Fig. 4c).

From the experimental data clearly, it appears that above a certain droplet volume, the droplet starts to freeze (Fig. 4a). Thus, any factor able to delay or avoid the increase in size of contact area is expected to be also able to delay or avoid droplet freezing. Photographs of the freezing droplets with a volume of $5 \mu \mathrm{L}$ on an uncoated glass substrate and the coated surfaces are shown in Fig. 5. The contact area between the FAS13 and FAS17 coating surfaces and water droplet was smaller than that of DTMS. The water contact angles of droplets before and after freezing were correlated. The contact angle freezing droplet on DTMS was larger than that of FAS13 and FAS17.
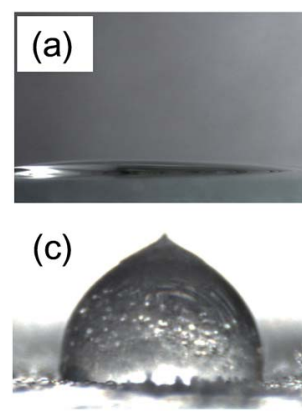

(d)
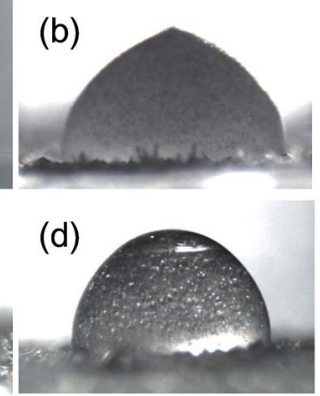

Fig. 5 Photographic images of freezing droplets on (a) a glass substrate, and surfaces coated with (b) DTMS, (c) FAS13, and (d) FAS17. Peltier element in air with a relative humidity of about $51 \%$ and temperature of $24{ }^{\circ} \mathrm{C}$. A water droplet put on Peltier element set at -8 $\pm 1^{\circ} \mathrm{C}$ for $20 \mathrm{~min}$. The droplet volume is $5 \mu \mathrm{L}$.

\section{Nucleation free energy barrier}

The nucleation free energy barrier ${ }^{26,40}$ of a coated surface is higher than that of an uncoated glass substrate. Based on classic nucleation theory, the nucleation free energy barrier can be calculated by the following equations. ${ }^{40,58}$

$$
\begin{gathered}
\Delta G=\left(\frac{-4 \pi r^{3} \Delta \mu_{\mathrm{v}}}{3}+4 \pi r^{2} \gamma_{\mathrm{nl}}\right) f(\theta) \\
f(\theta)=\frac{(1-\cos \theta)^{2}(2+\cos \theta)}{4}
\end{gathered}
$$

where $\Delta \mu_{\mathrm{v}}$ is the difference in the change of Gibb's free energy per unit volume between ice and liquid water, $\gamma_{\mathrm{nl}}$ is the interfacial tension between ice nucleation and liquid, and $f(\theta)$ is a geometric factor. There is a strong correlation between $\theta$ and water contact angle. According to these equations, $\Delta G$ is directly proportional to $f(\theta)$. This means that the larger $\theta$, the higher the free energy barrier. The plot of $f(\theta)$ in Fig. S17† reveals the $f(\theta)$ increases monotonically with $\theta$. Therefore, the increase of contact angle between ice nucleation and the substrate hinders ice crystallization. ${ }^{40,58,59}$

\section{Polarity and polarizability of fluorine atoms}

The mobility of water molecules decreased on the coated surfaces particularly with the fluorinated coating because of the polarity and low polarizability of the fluorine atoms of FAS13 and FAS17. ${ }^{\mathbf{5 4 6 3}}$ The existence of interactions between water molecules and fluorocarbon surfaces has been predicted by computational energy calculations presented in several studies. Here, we calculated the Hückel charge of long alkyl-chain and fluorocarbon-chain, and water molecules using the extended Hückel method (Fig. 6a, b, S18 and Table S4 $\dagger$ ). ${ }^{60}$ Fluorocarbon molecules have a polarizability that is about ten times higher than that of alkyl molecules (Table $\mathrm{S} 4 \dagger$ ). Schematic diagrams of atom properties also indicated the higher Hückel charge of fluorocarbons compared with that of alkyl chains (Fig. 6a and b). In per-fluorurate compounds, due to symmetry of the polarity vectors that perfectly compensate, the effective polarity of the whole molecule is close to zero. On the contrary, FAS13 and FAS17, that are not completely perfluorurated, are polar because of the local non complete compensation of the $\mathrm{C}-\mathrm{F}$ dipole moment. ${ }^{\mathbf{6 1}}$

It is considered that water molecules interact with FAS13 and FAS17 only because of this polarity, indeed water molecules are polar due to their oxygen atom. Therefore, we considered that water molecules are attracted by those coatings through polar interactions, also causing the mobility of water molecules to decrease. Water molecules form a hexagonal structure when liquid water turns into ice crystals; therefore, liquid-phase water molecules need to move to form ice crystals. The low mobility of water molecules on the FAS13and FAS17-coated surfaces prevents the formation of ice crystals from the liquid phase. ${ }^{54}$ To confirm the molecular interactions between the surfaces and water, we measured their advancing contact angle $\left(\theta_{\mathrm{Adv}}\right)$, receding contact angle $\left(\theta_{\text {Rec }}\right)$, and contact angle hysteresis $(\Delta \theta)$, the results are 


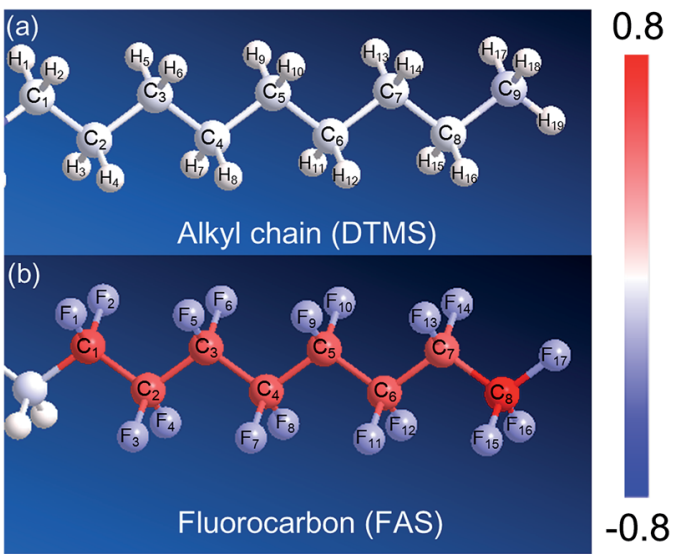

(c) Advancing contact angle $\quad$ Receding contact angle $\diamond$ Contact angle hysteresis

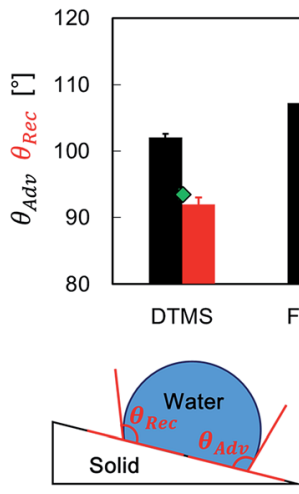

Decyltrimethoxysilane

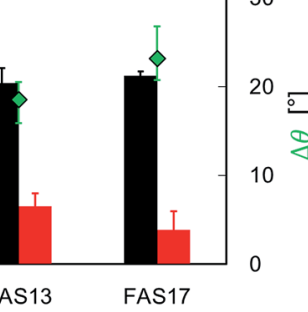

Stick!!

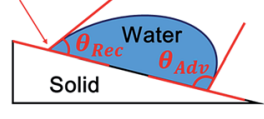

Fluoroalkylsilane

Fig. 6 Schematic diagrams of Hückel charge distribution in an (a) alkyl chain and (b) fluorocarbon. Red indicates positive charge and blue indicates negative charge; the color intensity is proportional to the charge strength. (c) Comparison of advancing contact angle $\left(\theta_{\text {Adv }}\right)$, receding contact angle $\left(\theta_{\text {Rec }}\right)$, and contact angle hysteresis $\left(\Delta \theta=\theta_{\mathrm{Adv}}-\theta_{\mathrm{Rec}}\right)$ of the three coating surface.

presented in Fig. 6c. We found that $\theta_{\text {Rec }}$ and $\Delta \theta$ of water on the surfaces coated with fluoroalkylsilanes were higher than those of the surface coated with DTMS. This behaviour can be explained in terms of different polarity and polarizability of fluorinated coating surface (FAS17 and FAS13) in comparison with the hydrogenated coating (DTMS). The interactions between these fluorinated coating surface and water are mostly residual permanent dipole-dipole bonds due to the high electronegativity and low polarizability of fluorine atom as well as to the permanent water polarity. On the contrary, the interaction between the hydrogenated coating and water are mostly due the high polarizability of the $\mathrm{C}-\mathrm{H}$ bond rather than to permanent dipole interaction. ${ }^{62}$ Therefore, we considered dipole-dipole the interactions between water with the fluorinated coating surface based on FAS17 and FAS13 and dipoleinduce dipole the interactions between water and the hydrogenated coating based on DTMS. The dipole-induced dipole interaction are normally very low and decrease dramatically with the distance, ${ }^{63}$ this promotes the mobility of water droplets on DTMS compared to water droplets on FAS17 and

FAS13, due to a further decrease in energy barrier to water droplets mobility on DTMS as shown in Fig. 6c. Furthermore, $\theta_{\text {Rec }}$ and $\Delta \theta$ of water on the surface coated with FAS17 were higher than those of one modified with FAS13. Therefore, water droplets interact more permanently with fluoroalkylsilanes compared with DTMS, and a higher content of fluorine atoms further strengthens this interaction. These findings support the experimental result that the ability of the FAS17 coating to prevent frost formation is greater than those of the FAS13 and DTMS coatings.

\section{Conclusion}

The smooth surface coatings using three types of low-surfaceenergy coupling agents with high molecular chain mobility were fabricated. These surfaces possessed RMS roughnesses lower than $1 \mathrm{~nm}$ and were oleophobic. The coating surfaces showed sliding angles of water of about $20^{\circ}$ and stearic acid and hexadecane of about $10^{\circ}$. The fluorine surface concentration of the FAS17 coating was about 1.3 times larger than that of the FAS13 coating. In addition, the surface energies of these coating surfaces decreased from DTMS $\left(23.86 \mathrm{mN} \mathrm{m}^{-1}\right)$ to FAS13 $\left(15.95 \mathrm{mN} \mathrm{m}^{-1}\right)$ to FAS17 $\left(15.08 \mathrm{mN} \mathrm{m}^{-1}\right)$. These coating surfaces delayed frost formation compared with that of an uncoated surface. The fluorocarbon-based coating surfaces delayed frost formation until $-6{ }^{\circ} \mathrm{C}$, and thus were more effective than the alkyl chain-based coating. In particular, the FAS17 coating effectively delayed frost formation. This indicates that a higher content of fluorine rate increases anti-frosting ability. This result was attributed to the antiwetting property of fluorocarbons, which increased the ice nucleation free energy barrier and promoted coalesce of condensed water droplets to decrease the solid-liquid contact area. Furthermore, the strong Hückel charge of fluorocarbons, which is about ten times higher than that of alkyl molecules, prevents movement of water molecules to form ice because of the peculiar and more permanent interaction of fluorocarbons with water molecules compared to the low dipole-induced dipole interaction between water and hydrocarbon. Surface chemical modification plays an important role in surface physical properties such as anti-wetting, surface tension and polarity, as well as anti-frosting behavior. In the future, we intend to consider surfaces coated with various low-surfaceenergy agents that alter surface physical properties to find the coatings that enhance anti-frosting ability. Our study revealed the influence of surface properties on anti-frosting ability, and provides guidelines for the further development of anti-frosting coating technology.

\section{Author contributions}

T. Moriya conceived and carried out the experiments. T. Moriya, K. M. and K. S. designed equipment and analyzed the data. T. Moriya, K. M., M. T. H. T. and T. Matsubayashi wrote the paper. M. T. provided experimental support and support in data analysis. W. N. commented on the manuscript. S. S. supervised the project and commented on the manuscript. 


\section{Acknowledgements}

We are grateful to Dr Kouji Fujimoto whose comments and suggestions were greatly valuable throughout our study. We are indebted to Dr Yoshio Hotta, whose relevant comments were an enormous help. We also thank Prof. Sae-Hoon Kim of Gangneung-Wonju National University for very useful discussions. This work was partially supported by JSPS KAKENHI Grant Number JP26420710.

\section{References}

1 J. Lv, Y. Song, L. Jiang and J. Wang, ACS Nano, 2014, 8, 31523169.

2 M. J. Kreder, J. Alvarenga, P. Kim and J. Aizenberg, Nature Reviews Materials, 2016, 1, 15003.

3 S. Nishimoto and B. Bhushan, RSC Adv., 2013, 3, 671-690.

4 J. L. Laforte, M. A. Allaire and J. Laflamme, Atmos. Res., 1998, 46, 143-158.

5 O. Gohardani, J. Aeronaut. Aero. Eng., 2012, 1, 116.

6 S. K. Thomas, R. P. Cassoni and C. D. MacArthur, J. Aircr., 1996, 33, 841-854.

7 M. Farzaneh, C. Volat and A. Leblond, in Anti-icing and deicing techniques for overhead lines, Springer, Netherlands, 2008, ch. 6, pp. 229-268.

8 D. D. Williams, N. E. Williams and Y. Cao, Water Res., 2000, 34, 127-138.

9 X. Sun, V. G. Damle, S. Liu and K. Rykaczewski, Adv. Mater. Interfaces, 2015, 2, 1400479.

10 X. Yin, Y. Zhang, D. Wang, Z. Liu, Y. Liu, X. Pei, B. Yu and F. Zhou, Adv. Funct. Mater., 2015, 25, 4237-4245.

11 S. Kiruthika, R. Gupta and G. U. Kulkarni, RSC Adv., 2014, 4, 49745-49751.

12 S. Ozbay, C. Yuceel and H. Y. Erbil, ACS Appl. Mater. Interfaces, 2015, 7, 22067-22077.

13 T. Sun, L. Feng, X. Gao and L. Jiang, Acc. Chem. Res., 2005, 38, 644-652.

14 K. Sasaki, M. Tenjimbayashi, K. Manabe and S. Shiratori, ACS Appl. Mater. Interfaces, 2016, 8, 651-659.

15 T. Matsubayashi, M. Tenjimbayashi, K. Manabe, K. H. Kyung, B. Ding and S. Shiratori, RSC Adv., 2016, 6, 15877-15883.

16 N. Yokoi, K. Manabe, M. Tenjimbayashi and S. Shiratori, ACS Appl. Mater. Interfaces, 2015, 7, 4809-4816.

17 F. Zhao, X. Wang, B. Ding, J. Lin, J. Hu, Y. Si, J. Yu and G. Sun, RSC Adv., 2011, 1, 1482-1488.

18 S. Talaeemashhadi, M. Sansotera, C. Gambarotti, A. Famulari, C. L. Bianchi, P. A. Guarda and W. Navarrini, Carbon, 2013, 59, 150-159.

19 M. He, J. Wang, H. Li and Y. Song, Soft Matter, 2011, 7, 39934000.

20 Y. Shen, H. Tao, S. Chen, L. Zhu, T. Wang and J. Tao, RSC Adv., 2015, 5, 1666-1672.

21 A. J. Meuler, J. D. Smith, K. K. Varanasi, J. M. Mabry, G. H. McKinley and R. E. Cohen, ACS Appl. Mater. Interfaces, 2010, 2, 3100-3110.
22 L. Wang, M. Wen, M. Zhang, L. Jiang and Y. Zheng, J. Mater. Chem. A, 2014, 2, 3312-3316.

23 T. M. Schutzius, S. Jung, T. Maitra, G. Graeber, M. Köhme and D. Poulikakos, Nature, 2015, 527, 82-85.

24 T. Maitra, M. K. Tiwari, C. Antonini, P. Schoch, S. Jung, P. Eberle and D. Poulikakos, Nano Lett., 2013, 14, 172-182.

25 J. Liu, H. Guo, B. Zhang, S. Qiao, M. Shao, X. Zhang, X. Feng, Q. Li, Y. Song, L. Jiang and J. Wang, Angew. Chem., Int. Ed., 2016, 55, 4265-4269.

26 K. K. Varanasi, T. Deng, J. D. Smith, M. Hsu and N. Bhate, Appl. Phys. Lett., 2010, 97, 234102.

27 J. Feng, Y. Pang, Z. Qin, R. Ma and S. Yao, ACS Appl. Mater. Interfaces, 2012, 4, 6618-6625.

28 Y. Sohn, D. Kim, S. Lee, M. Yin, J. Y. Song, W. Hwang, S. Park, H. Kim, Y. Ko and I. Han, J. Mater. Chem. A, 2014, 2, 1146511471.

29 S. A. Kulinich, S. Farhadi, K. Nose and X. W. Du, Langmuir, 2010, 27, 25-29.

30 T. S. Wong, S. H. Kang, S. K. Tang, E. J. Smythe, B. D. Hatton, A. Grinthal and J. Aizenberg, Nature, 2011, 477, 443-447.

31 I. Okada and S. Shiratori, ACS Appl. Mater. Interfaces, 2014, 6, 1502-1508.

32 K. Manabe, K. H. Kyung and S. Shiratori, ACS Appl. Mater. Interfaces, 2015, 7, 4763-4771.

33 T. Song, Q. Liu, M. Zhang, R. Chen, K. Takahashi, X. Jing, L. Liu and J. Wang, RSC Adv., 2015, 5, 70080-70085.

34 P. Kim, T. S. Wong, J. Alvarenga, M. J. Kreder, W. E. AdornoMartinez and J. Aizenberg, ACS Nano, 2012, 6, 6569-6577.

35 P. W. Wilson, W. Lu, H. Xu, P. Kim, M. J. Kreder, J. Alvarenga and J. Aizenberg, Phys. Chem. Chem. Phys., 2013, 15, 581-585.

36 K. Manabe, S. Nishizawa, K. H. Kyung and S. Shiratori, ACS Appl. Mater. Interfaces, 2014, 6, 13985-13993.

37 L. Chen, A. Geissler, E. Bonaccurso and K. Zhang, ACS Appl. Mater. Interfaces, 2014, 6, 6969-6976.

38 N. Vogel, R. A. Belisle, B. Hatton, T. S. Wong and J. Aizenberg, Nat. Commun., 2013, 4, 2176.

39 K. Rykaczewski, S. Anand, S. B. Subramanyam and K. K. Varanasi, Langmuir, 2013, 29, 5230-5238.

40 X. Zhan, Y. Yan, Q. Zhang and F. Chen, J. Mater. Chem. A, 2014, 2, 9390-9399.

41 Y. Tang, Q. Zhang, X. Zhan and F. Chen, Soft Matter, 2015, 11, 4540-4550.

42 Z. He, W. J. Xie, Z. Liu, G. Liu, Z. Wang, Y. Q. Gao and J. Wang, Sci. Adv., 2016, 2, e1600345.

43 H. Yang, C. Ma, K. Li, K. Liu, M. Loznik, R. Teeuwen, J. C. M. van Hest, X. Zhou, A. Herrmann and J. Wang, Adv. Mater., 2016, 28, 5008-5012.

44 C. Urata, D. F. Cheng, B. Masheder and A. Hozumi, RSC Adv., 2012, 2, 9805-9808.

45 C. Urata, B. Masheder, D. F. Cheng and A. Hozumi, Langmuir, 2012, 28, 17681-17689.

46 C. Urata, B. Masheder, D. F. Cheng and A. Hozumi, Chem. Commun., 2013, 49, 3318-3320.

47 C. Urata, B. Masheder, D. F. Cheng, D. F. Miranda, G. J. Dunderdale, T. Miyamae and A. Hozumi, Langmuir, 2014, 30, 4049-4055. 
48 Q. Zhang, M. He, J. Chen, J. Wang, Y. Song and L. Jiang, Chem. Commun., 2013, 49, 4516-4518.

49 X. Chen, J. Wu, R. Ma, M. Hua, N. Koratkar, S. Yao and Z. Wang, Adv. Funct. Mater., 2011, 21, 4617-4623.

50 Y. Wang, M. Li, T. Lv, Q. Wang, Q. Chen and J. Ding, J. Mater. Chem. A, 2015, 3, 4967-4975.

51 Q. Fu, X. Wu, D. Kumar, J. W. C. Ho, P. D. Kanhere, N. Srikanth, E. Liu, P. Wilson and Z. Chen, ACS Appl. Mater. Interfaces, 2014, 6, 20685-20692.

52 A. P. Esser-Kahn, V. Trang and M. B. Francis, J. Am. Chem. Soc., 2010, 132, 13264-13269.

53 X. Li, K. Zhang, Y. Zhao, K. Zhu and X. Yuan, RSC Adv., 2015, 5, 90578-90587.

54 S. Suzuki, A. Nakajima, N. Yoshida, M. Sakai, A. Hashimoto, Y. Kameshima and K. Okada, Chem. Phys. Lett., 2007, 445, 37-41.

55 M. Sakai, J. H. Song, N. Yoshida, S. Suzuki, Y. Kameshima and A. Nakajima, Langmuir, 2006, 22, 4906-4909.
56 A. Hozumi, K. Ushiyama, H. Sugimura and O. Takai, Langmuir, 1999, 15, 7600-7604.

57 C. J. van Oss, J. Mol. Recognit., 2003, 16, 177-190.

58 G. Hass and R. E. Thun, in Physics of Thin Films, Academic Press, New York and London, 1967, vol. 4, pp. 99-104.

59 Y. Zhang, E. Anim-Danso, S. Bekele and A. Dhinojwala, ACS Appl. Mater. Interfaces, 2016, 8, 17583-17590.

60 R. Hoffmann, J. Phys. Chem., 1963, 39, 1397-1412.

61 P. Kirsh, in Modern Fluoroorganic Chemistry. Synthesis, Reactivity, Applications, Wiley-VCH, Weinheim, 2004, ch. 1, pp. 9-13.

62 B. Smart, in Organofluorine Chemistry: Principles and Commercial Applications, ed. R. E. Banks, et al., Plenum Press, New York, 1994, ch. 3, pp. 57-88.

63 T. Blythe and D. Bloor, in Electrical Property of Polymers, Cambridge University Press, Cambridge, 2nd edn, 2005, ch. 2, pp. 31-34. 